Arthroskopie 2020 $33: 119$

https://doi.org/10.1007/s00142-019-00322-9

(C) Springer Medizin Verlag GmbH, ein Teil von Springer Nature 2019

\author{
M. Flury' $\cdot$ A. Voss ${ }^{2}$ \\ 'FMH Orthopädie \& Traumatologie, IN MOTION, Wallisellen, Schweiz \\ ${ }^{2}$ Sporthopaedicum Straubing-Regensburg \& Klinik für Unfallchirurgie, Universitätsklinikum Regensburg, \\ Regensburg, Deutschland
}

\title{
Komplikationsmanagement der oberen Extremität
}

Liebe Leserinnen und Leser,

Im Verlauf der letzten Jahre wurden im Bereich der Schulter- und Ellbogenchirurgie auf allen Ebenen sehr viele neue Erkenntnisse gewonnen, begonnen mit der Anatomie über die Biomechanik bis hin zu den therapeutischen Optionen. Dadurch konnten die Resultate verbessert und vielfach das Komplikationsrisiko reduziert werden. Trotzdem wird auch in Zukunft jeder operativ tätige Arzt mit Komplikationen konfrontiert werden.

Diese meist komplexen Situationen bedürfen einer maßgeschneiderten, patientenadaptierten Lösung. Das beginnt bei der korrekten Aufklärung vor der Intervention über die Erkennung und Diagnose (Stichwort: „Ich sehe nur das, was ich kenne") bis hin zu den Therapieoptionen.

\section{》) Die psychologischen Aus- wirkungen einer Komplikation dürfen nicht unterschätzt werden}

Dabei muss bedacht werden, dass jede Komplikation für den Patienten schwierig, gelegentlich sogar katastrophal ist. Daher dürfen die psychologischen Auswirkungen einer Komplikation sowohl für den behandelnden Arzt als auch für den Patienten nicht unterschätzt werden. Der Arzt ist mit dem Resultat unzufrieden und spürt die Erwartungshaltung des Patienten. Der Patient ist verunsichert und frustriert. In dieser Situation hilft nur eine sehr intensive Patientenbetreuung. Diese basiert einerseits darauf, dass man die Komplikation erkennt, akzeptiert und vor allem offen und ehrlich darüber kommuniziert. Andererseits ist eine fundierte Patientenberatung sehr wich- tig, um die individuell beste Lösung zu finden.

Diese Leitthemenausgabe soll Ihnen helfen, die möglichen Komplikationen besser $\mathrm{zu}$ verstehen und dadurch das Management der Komplikation zu verbessern. Damit lassen sich die aktuell immer häufiger diskutierten medikolegalen Auswirkungen reduzieren. Die Erfahrung zeigt, dass die meisten Patienten bereit sind, nahezu jede Komplikation zu akzeptieren, solange sie sich ernstgenommen und individuell gut betreut fühlen.

Deshalb sind wir froh darüber, dass wir eine Gruppe renommierter Experten gewinnen konnten, die ihre Erfahrungen und Vorgehensweisen in diesen schwierigen Situationen mit uns teilen. Dies soll Ihnen helfen, Ihr persönliches Komplikationsmanagement weiterzuentwickeln und $\mathrm{zu}$ verbessern, um das bestmögliche Resultat nach einer Komplikation zu erreichen. Denn die Qualität eines Chirurgen misst sich nicht zuletzt daran, wie er mit den Komplikationen seiner Arbeit umgeht und wie er sie zu behandeln weiß!

\section{Korrespondenzadresse}

Dr. med. M. Flury

FMH Orthopädie \& Traumatologie, IN MOTION 8304 Wallisellen, Schweiz matthias.flury@in-motion.ch

\section{PD Dr. med. A. Voss}

Sporthopaedicum Straubing-Regensburg \&

Klinik für Unfallchirurgie, Universitätsklinikum Regensburg Franz-Josef-Strauss-Allee 11, 93053 Regensburg, Deutschland

a.voss@tum.de

Interessenkonflikt. M. Flury hat einen Beratervertrag und bezieht Royalties von der Firma Arthrex GmbH München. A. Voss gibt an, dass kein Interessenkonflikt besteht. 